\title{
SPIN Protocol in Wireless Sensor Network
}

\author{
${ }^{1}$ Ankit Gaur \\ Department of Computer Science, \\ CDAC, NOIDA, \\ New Delhi, India
}

\author{
${ }^{2}$ Ms. Rosy Verma \\ Department of Computer Science, \\ CDAC, NOIDA, \\ New Delhi, India
}

\begin{abstract}
Wireless Sensor network has become the most researched area due to its huge importance in the area of IOT. In this technique the nodes sends only the useful information to the base station. This increase the communicational overhead and affects the network's lifetime. Since energy conservation is the key issue in WSN, proper data dissemination should be incorporated in order to save energy. The main aim is to route the data on the network in an energy efficient manner, so that network lifetime is enhanced. In this paper, we present Sensor Protocol for Information via Negotiation where both energy value of sensor node as well as hop count is considered for selection among the neighbor node to whom data is to be forwarded. Through this technique the data is send to all the nodes in the network. The issues focused here are minimizing of energy consumption and maximizing the network lifetime of the Wireless Sensor Network.
\end{abstract}

Keywords---Wireless Sensor Network, Flooding, LEACH, SPIN, $A D V, R E Q, D A T A$

\section{INTRODUCTION}

Wireless Sensor Network consists of sensor nodes which are scattered over a region for monitoring the various physical phenomena like temperature, humidity, vibrations and so on. The energy constraint problem which arises in wireless sensor network is solved by using SPIN protocol. Previously LEACH protocol was implemented, which communicate by forming cluster head of sensor nodes but it is not energy efficient as SPIN protocol is. So we are implementing SPIN protocol in wireless sensor network to reserve the energy of the sensor nodes and increase the lifetime of the network. Nodes can deplete due to defected batteries and environment factors and one node can damage the whole network. Node failure, node mobility and environmental obstructions can cause a high degree of mobility in WSN. WSN may have large number of sensor nodes. All nodes are not of same computation power and it provides Redundancy.

\section{SPIN PROTOCOL}

It transfers all the useful data only from each node to every node in the network assuming that all the nodes in the network are Base Station. SPIN node uses three types of messages for communication.

1. ADV-It is used to advertise new data.

2. REQ- REQ is used to receive the actual data.

3. DATA- DATA is the actual message itself.

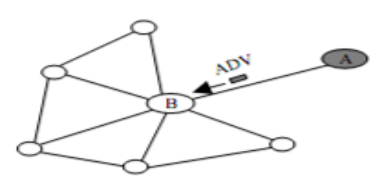

(a)

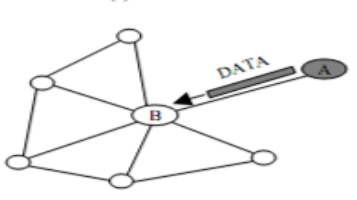

(c)

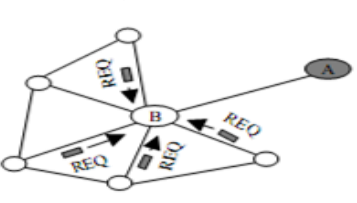

(e)

Fig 1. Working of $\operatorname{SPIN}^{[7]}$

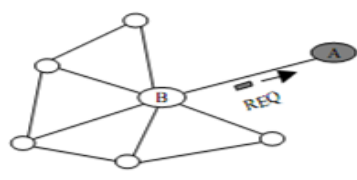

(b)

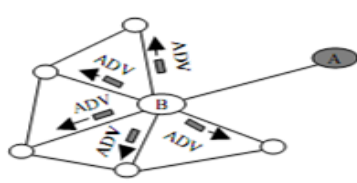

(d)

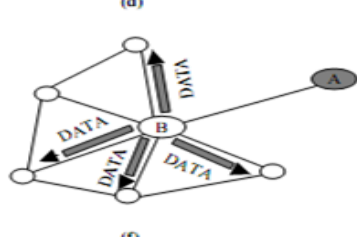

Working of SPIN protocol

1. Node A starts by Advertising its data to the node B.

2. Node B responds by sending a request to the node A.

3. As soon as node $B$ receives the request it sends the original data to its neighbor nodes and similarly the process goes on till all the nodes in the network receives the data.

4. This process continues till all the nodes receives the data.

\section{ADVANTAGES OF SPIN}

SPIN solves the problem of Implosion, Overlap and thus achieve a lot of energy efficiency.

\section{Implosion}

Node A starts by flooding the data to its two neighbours i.e $\mathrm{B}$ and $\mathrm{C}$. These nodes store the data received from $\mathrm{A}$ and send a copy of it to their neighbor D. The protocol thus wastes energy and bandwidth by sending one extra copy of A to D. 


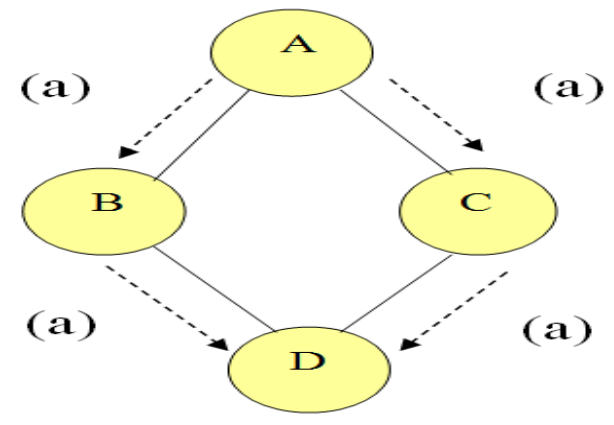

Fig 2. Implosion ${ }^{[7]}$

\section{Overlap}

The sensor area of node A and node B are overlapping and the neighbour node of $\mathrm{A}$ and $\mathrm{B}$ are same therefore node $\mathrm{C}$ receives 2 copies of $\mathrm{Y}$ and energy is wasted.

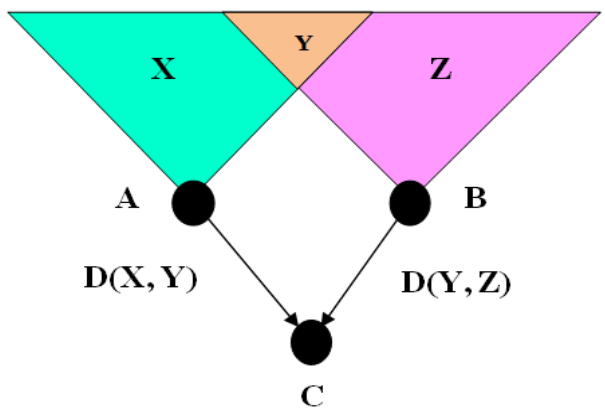

Fig 3. Overlap ${ }^{[7]}$

IV. COMPONENTS OF WSN

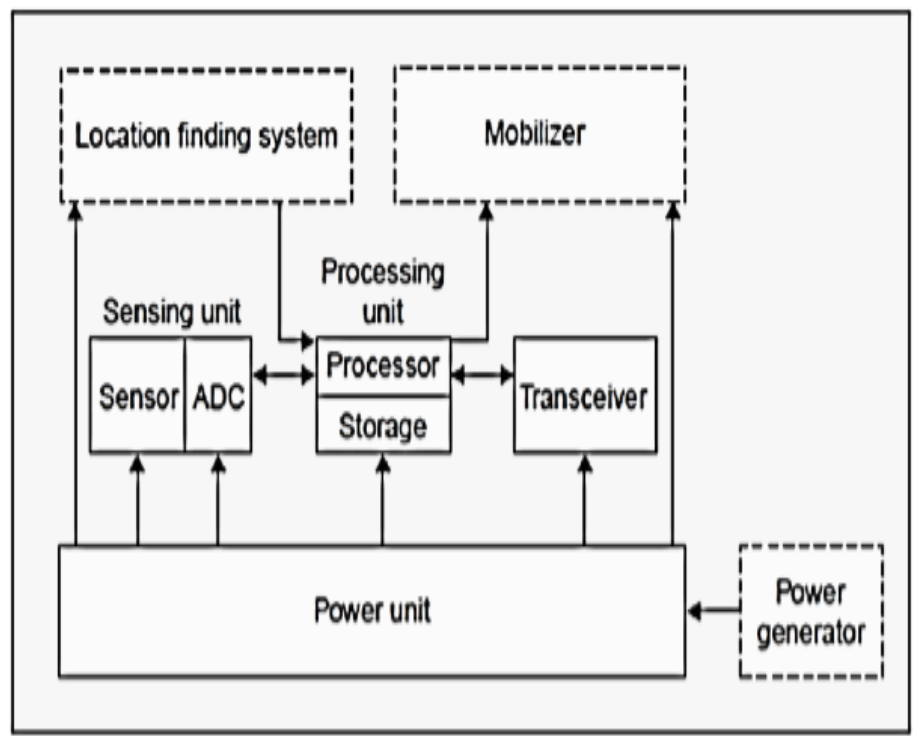

Fig 4. Components of Sensor node ${ }^{[10]}$

1. Sensing Unit:- Sensing units are made up of two units Sensor and ADC. ADC transforms Analog signal to Digital signal and transfer the information to the processing unit.

2. Processing Unit:- It processes the sensor nodes and store the information which is send by the sensing unit and then send it back to the transceiver.

3. Transceiver:- Transceiver connects the node to the network.
4. Power Unit:- Power Units consists of solar cells which sends the power to the whole network.

5. Power Generator:- It gives power to the whole WSN.

\section{PROTOCOL STACK OF WSN}

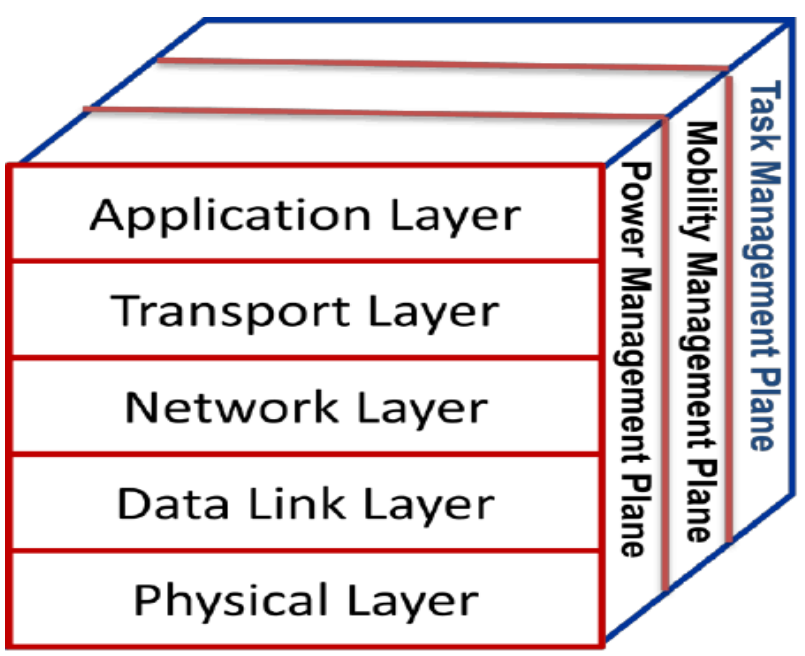

Fig 5. Protocol stack of WSN ${ }^{[10]}$

1. Application layer:- Application layer uses many types of software which can be made and implement on this layer.

2. Transport layer:- It provide need in maintaining and processing of the data.

3. Network layer:- It provide routing of the data by end to end delivery layer.

4. Data link layer:- It uses the Mac protocol which can reduce collision with the other node.

5. Physical layer:- It is used for simple broadcasting and transmission techniques.

6. Power, Mobility and Task Management planes helps in coordinating Sensing data of the WSN and lowers the overall power consumption.

\section{PROBLEM STATEMENT}

1. Sensor nodes has a limitation for power supply, as it is provided by the battery.

2. So we need to work in order to minimize the energy consumption of the sensor nodes.

\section{DISADVANTAGES OF SPIN}

1.The sending of data towards the sink node from the source node takes very long time.

2.If a node has more computation power then it will consume more energy as compared to other node in the network.

3.If a node is used many times then it will lose energy early then the other nodes in the network.

4.If a node is sitting idle then its energy will be reduced without transmission of data. 


\section{PROPOSED SOLUTION}

1. The Initial Phase: - I have read about my topic from the papers like IEEE, Springer etc which has helped me a lot to understand and implement my project.

2. The Implementation Phase: Matlab software will be used for the implementation of my project using SPIN algorithm.

3. The Testing Phase: At last we will conduct a analysis for the various network parameters.

\begin{tabular}{|l|l|}
\multicolumn{2}{l}{ IX. RESULTS } \\
\hline Area & $100 \mathrm{~m}^{*} 100 \mathrm{~m}$ \\
\hline Number of Nodes & 100 \\
\hline Transmit(ETX)andReceive(ERX) & $50 \mathrm{~nJ} / \mathrm{bit}$ \\
\hline Transmit Amplifier(E amp) & $100 \mathrm{pJ} / \mathrm{bit} / \mathrm{m}^{\wedge} 2$ \\
\hline Initial Energy of Sensor node(Eo) & $0.5 \mathrm{~J}$ \\
\hline Control Message Size & $100 \mathrm{bits}$ \\
\hline Data Message Size & $2000 \mathrm{bits}$ \\
\hline Threshold & $0.005 \mathrm{~J}$ \\
\hline Transmission Range & $20 \mathrm{~m}$ \\
\hline
\end{tabular}

Table 1. Simulation parameters

1. An area of $100 \mathrm{~m} * 100 \mathrm{~m}$ is taken where 100 sensor nodes are randomly distributed on it.

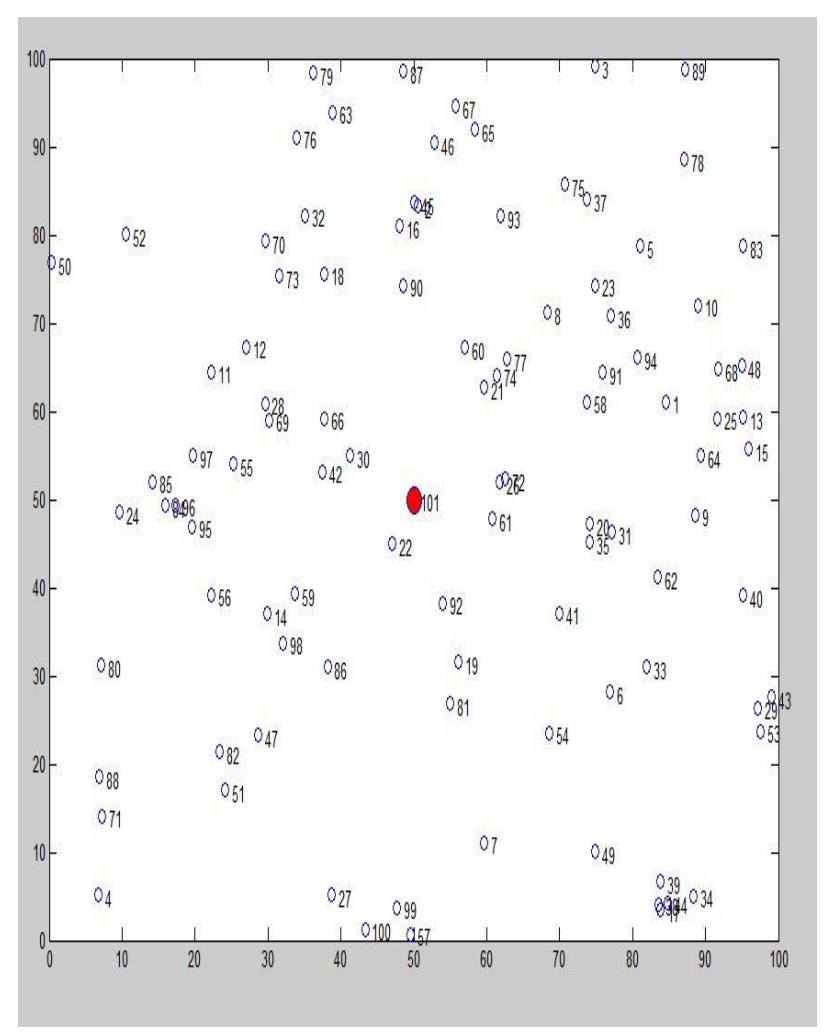

2. Graph of Live nodes verses No. of Round: This graph shows number of live nodes in the network.

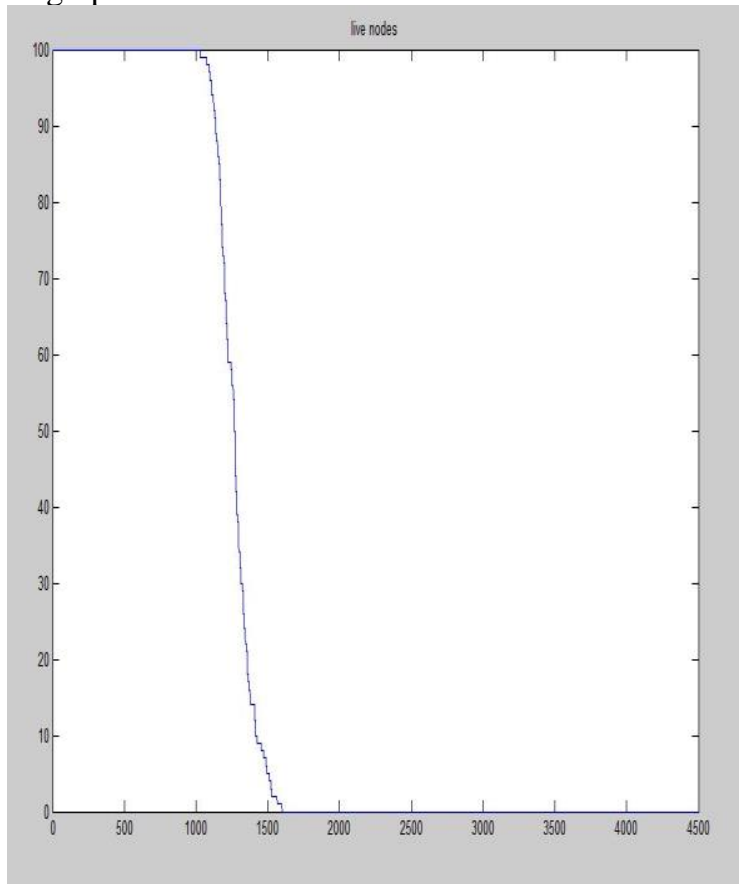

3 Graph of Dead Node verses No. of Round: This graph shows Number of Dead nodes in the network.

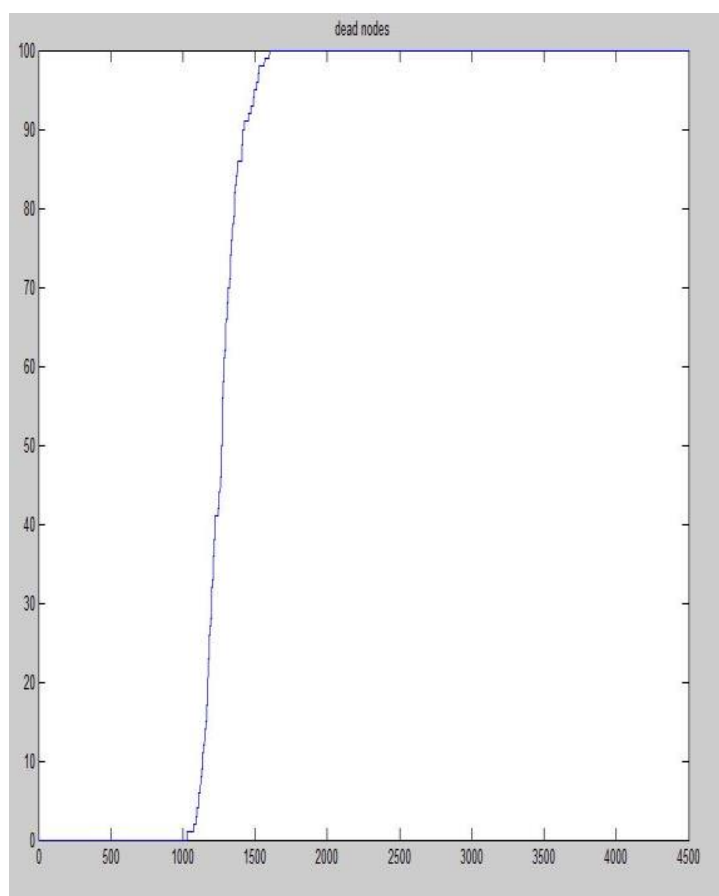


4. Graph showing Residual Energy versus No of Rounds In ISPIN, SPIN, MSPIN, Flooding.

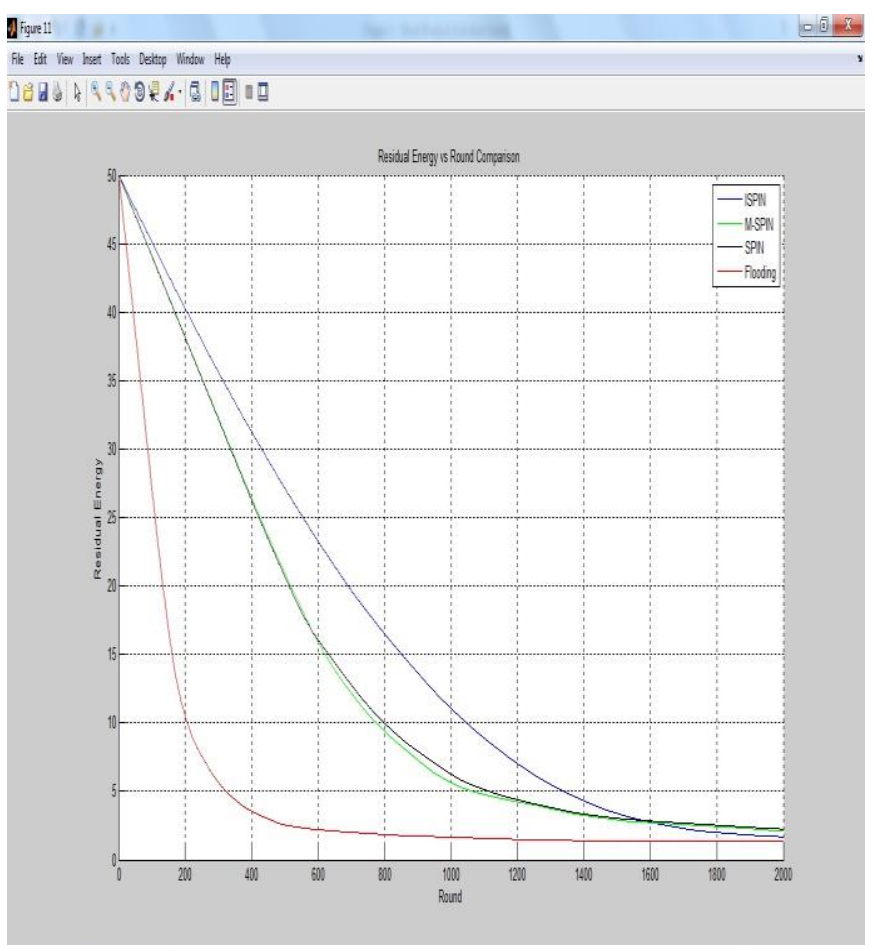

5. Graph showing Dead nodes versus No of Rounds in ISPIN, SPIN, MSPIN, Flooding.

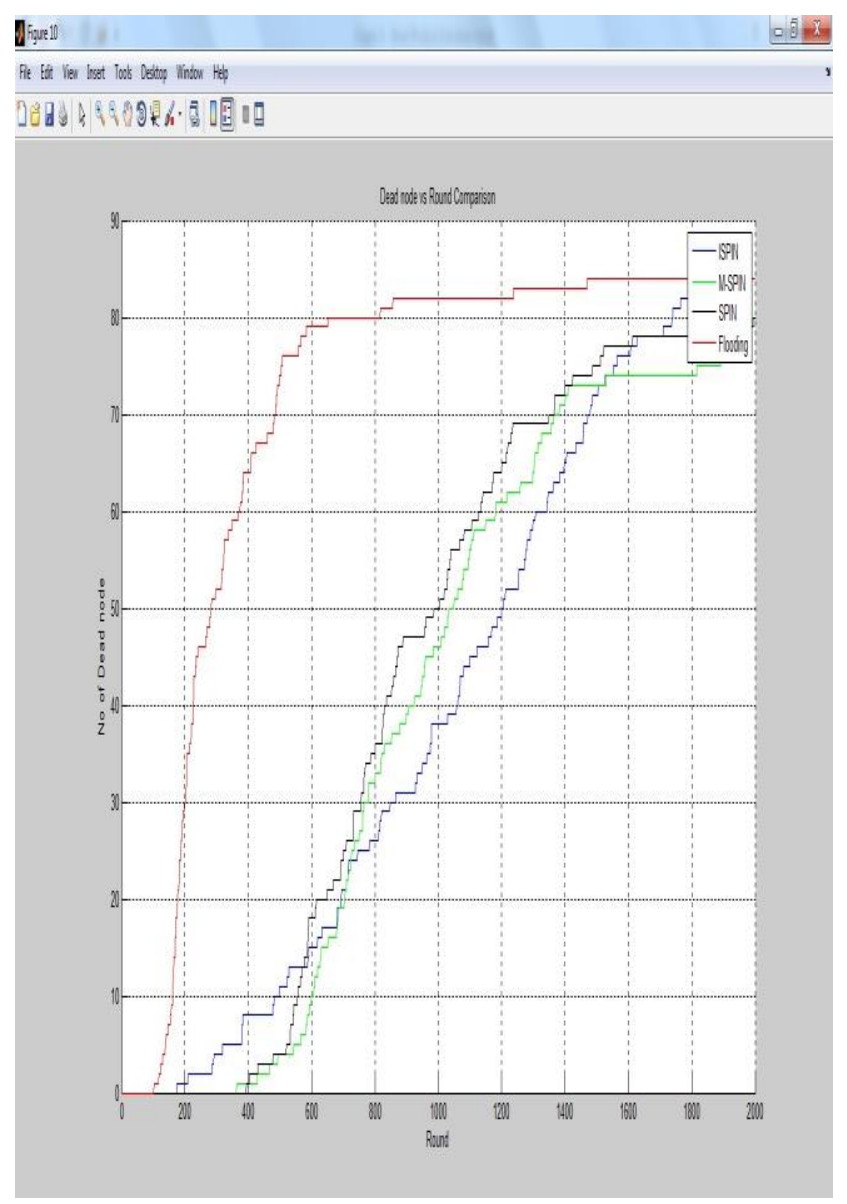

\section{CONCLUSION}

The paper entitled SPIN Protocol in Wireless Sensor Networks has been studied in detail. This paper contains introduction about the Wireless Sensor Network, SPIN protocol, components of WSN, Protocol Stack of WSN, basic concepts about WSN. This research mainly focuses on efficient routing algorithm in Wireless Sensor Network. The aim is to minimize the energy consumption of the sensor node and enhance the lifetime of the network. For this an efficient routing algorithm SPIN is proposed. In WSN, the routing protocols are used to increase the life cycle of the network and to decrease the consumption energy. The basis of our proposed protocol is the selection of minimum path by using minimum spanning tree concept to send the data from the source to the destination with minimum cost, minimum time and minimum path. To select the minimum path we have taken both residual energy and the hop count. The proposed protocol is implemented and compared with flooding. The proposed protocol shows a better performance in terms of residual energy and number of dead nodes.

\section{REFERENCES}

[1] Q. Wang, D. Lin, and Z. Zhang, "An Energy-Efficient Compressive Sensing-Based Clustering Routing Protocol for WSN," in IEEE Sensors Journal, vol. 19, no. 10, pp. 3950-3960, 2019.

[2] Jabbar S, Ahmad M, and Ahmad S.H, "A Novel Energy-Aware Design for Clustered Wireless Sensor Networks," in Recent Trends and Advances in Wireless sensor Networks, pp. 119-127, 2019.

[3] Y. Yuan, W. Liu, and H. Song, "Compressive Sensing-Based Clustering Joint Annular Routing Data Gathering Scheme for Wireless Sensor Networks," in IEEE Access, vol. 7, pp. 114639$114658,2019$.

[4] T. M. Behera, S. K. Mohapatra, and A. H. Gandomi, "Residual Energy-Based Cluster-Head Selection in WSN," in IEEE Internet of Things Journal, vol. 6, pp. 5132-5139, 2019.

[5] N. Wang and J. Li, "Shortest Path Routing with Risk Control for Compromised Wireless Sensor Networks," in IEEE Access, vol. 7, pp. 19303-19311, 2019.

[6] Y. Zhang, X. Zhang, and Y. Liu, "Energy-Efficient Multilevel Heterogeneous Routing Protocol for Wireless Sensor Networks," in IEEE Access, vol. 7, pp. 55873-55884, 2019.

[7] Kulik, J., Heinzelman, W. \& Balakrishnan, H. "Negotiation-Based Protocols for Disseminating Information in Wireless Sensor Networks," in IEEE Communication Magazine, pp. 169-185, 2002.

[8] Z. Rehena, S. Roy and N. Mukherjee, "A Modified SPIN for Wireless Sensor Networks," Third International Conference on Communication Systems and Networks, pp. 1-4, 2011.

[9] Luwei Jing, Feng Liu and Yuling Li, "Energy Saving Routing Algorithm based on SPIN Protocol in WSN," International Conference on Image Analysis and Signal Processing, pp. 416-419, 2011.

[10] Akyildiz,I.F.; Weilian Su; Sankarasubramaniam,Y.; Cayirci,E., "A Survey on Sensor Networks, A survey on sensor networks," Communications Magazine, IEEE Volume: 40, Issue: 8, 2002. 